\title{
Reflets
}

Revue d'intervention sociale et communautaire

\section{Qui sont les prestataires de l'aide sociale? Analyse des débats médiatiques entourant la réforme de l'aide sociale au Québec (2015-2016)}

\author{
Amélie Maugère et Élisabeth Greissler
}

Volume 25, numéro 1, printemps 2019

Actualité de la pauvreté : débats théoriques, défis pratiques

URI : https://id.erudit.org/iderudit/1064667ar

DOI : https://doi.org/10.7202/1064667ar

Aller au sommaire du numéro

Éditeur(s)

Reflets, Revue d'intervention sociale et communautaire

ISSN

1203-4576 (imprimé)

1712-8498 (numérique)

Découvrir la revue

Citer cet article

Maugère, A. \& Greissler, É. (2019). Qui sont les prestataires de l'aide sociale? Analyse des débats médiatiques entourant la réforme de l'aide sociale au Québec (2015-2016). Reflets, 25(1), 51-70. https://doi.org/10.7202/1064667ar
Résumé de l'article

Au Québec, la réforme de la politique en matière d'aide sociale, annoncée par le gouvernement libéral en janvier 2015 et adoptée définitivement par le parlement le 10 novembre 2016, a été très controversée. Pour spécifier les modèles normatifs en compétition qui émergent de cette controverse, nous avons collecté l'ensemble des articles d'opinion (tribunes politiques et militantes, tribunes d'éditorialistes, courrier des lecteurs) parus dans trois journaux de masse québécois entre 2015 et 2016. En nous inspirant de l'analyse cognitive des politiques publiques (Muller, 2005), nous avons identifié les principales références normatives et cognitives qui y sont mobilisées. Cette analyse met en évidence que les pro-réforme et les anti-réforme utilisent peu les mêmes références; en particulier, les prestataires y sont rarement décrits avec les mêmes caractéristiques. Cependant, les anti-réforme ont rarement proposé dans leur argumentaire un modèle normatif profondément alternatif à celui développé par le gouvernement.
Tous droits réservés @ Reflets, Revue d’intervention sociale et communautaire, 2019
Ce document est protégé par la loi sur le droit d'auteur. L'utilisation des services d'Érudit (y compris la reproduction) est assujettie à sa politique d'utilisation que vous pouvez consulter en ligne. 


\title{
Qui sont les prestataires de l'aide sociale? Analyse des débats médiatiques entourant la réforme de l'aide sociale au Québec (2015-2016)
}

\author{
Amélie Maugère \\ Professeure adjointe, École de travail social, Université de Montréal \\ Élisabeth Greissler \\ Professeure adjointe, École de travail social, Université de Montréal
}

\section{Résumé}

Au Québec, la réforme de la politique en matière d'aide sociale, annoncée par le gouvernement libéral en janvier 2015 et adoptée définitivement par le parlement le 10 novembre 2016, a été très controversée. Pour spécifier les modèles normatifs en compétition qui émergent de cette controverse, nous avons collecté l'ensemble des articles d'opinion (tribunes politiques et militantes, tribunes d'éditorialistes, courrier des lecteurs) parus dans trois journaux de masse québécois entre 2015 et 2016. En nous inspirant de l'analyse cognitive des politiques publiques (Muller, 2005), nous avons identifié les principales références normatives et cognitives qui y sont mobilisées. Cette analyse met en évidence que les pro-réforme et les anti-réforme utilisent peu les mêmes références; en particulier, les prestataires y sont rarement décrits avec les mêmes caractéristiques. Cependant, les anti-réforme ont rarement proposé dans leur argumentaire un modèle normatif profondément alternatif à celui développé par le gouvernement.

Mots clés : Aide sociale, prestataires, politique, action publique, réforme, militant, discours, approche cognitive, normativité, Québec, média, presse.

\section{Abstract}

In Quebec, the reform of social assistance policy, announced by the Liberal government in January 2015 and finally adopted by Parliament on November 10, 2016, was very controversial. To specify the normative models in competition that emerge from this controversy, we collected all the articles of opinion (political and activist forums, editorial 
forums, reader's letter) published in three Quebec mass newspapers between 2015 et 2016. We are modeling our approach on the cognitive policy analysis (Muller, 2005) in order to identify the main normative and cognitive references that are put forward. This analysis highlights that supporters and opponents rarely use the same references; especially, people benefiting from social assistance are rarely described with the same characteristics. The opponents, however, have rarely put forward in their argument an alternative to the normative model developed by the government.

Key words : social assistance, people on social assistance, policy, public action, reform, activist, discourse, cognitive policy analysis, normativity, Quebec, media, press

Au milieu des années 1990, les programmes d'aide sociale comme ceux de l'assurance emploi ont fait l'objet de plusieurs réformes néolibérales de l'État-providence (Merrien, Parchet et Kernen, 2005; Groulx, 2009). La première Loi de l'aide sociale du Québec est adoptée en 1969 et c'est en 1989 qu'a lieu une importante réforme qui, pour la première fois, propose une distinction entre les prestataires médicalement aptes et ceux inaptes au travail (Jetté et Bissonnette, 2012). Aujourd'hui, 3 catégories distinctes de prestataires sont identifiées : les personnes sans contrainte à l'emploi, celles ayant des contraintes temporaires et celles ayant des contraintes sévères.

Au Québec, la réforme de la politique de l'aide sociale (Loi sur l'aide aux personnes et aux familles), annoncée par le gouvernement libéral en janvier 2015 et adoptée définitivement par le parlement le 10 novembre 2016, a été très controversée. En novembre 2015, le dépôt du Projet de loi 70 a soulevé dans le milieu communautaire un vaste mouvement de contestation qui a mené, notamment dans la presse écrite, à une médiatisation d'une partie des enjeux y étant associés. Ces réformes ont généralement eu pour conséquence d'affaiblir la perspective "démarchandisante» de l'État-providence (Esping-Andersen, 1999). Véritables outils de gestion de la main d'œuvre, elles durcissent les conditions d'obtention de l'aide ou diminuent le montant des prestations financières (Fretel et collab., 2018). Ainsi, les prestataires ont moins la possibilité de vivre indépendamment du marché du travail. Au Québec, entre 1996 et 2016, le nombre de prestataires de l'aide sociale a diminué de moitié et l'on enregistre aujourd'hui son taux le plus bas depuis 40 ans. Se voulant un incitatif au travail, le niveau de couverture des besoins de base n'a cessé de diminuer pour les personnes vivant seules et n'atteint qu'environ $50 \%$ d'un revenu d'existence décent (IRIS, 2012; Collectif pour un Québec sans pauvreté, 2016). 
Au cœur de la controverse de la réforme annoncée en janvier 2015, figure la mesure prévoyant des sanctions faisant reculer la prestation de base mensuelle pour une personne seule de 623 \$ à 399 \$ en cas de manquements à des engagements du plan d'intégration à l'emploi. Cette mesure fait l'objet des plus vives réactions dans les articles d'opinion parus dans la presse au moment des débats parlementaires. Dans le cadre de cet article, et en nous intéressant à la manière dont les autrices ou auteurs d'articles d'opinion parus dans trois journaux de masse québécois avaient traité la thématique de l'aide sociale entre janvier 2015 et décembre 2016, nous mettrons à jour les valeurs et les formes de savoirs utilisées pour fonder auprès de l'opinion publique la légitimité de la réforme de la politique en matière d'aide sociale au Québec, ou au contraire, la saper. Après avoir exposé notre cadre conceptuel qui s'inspire de l'approche cognitive de l'action publique, nous présenterons la manière dont les personnes visées par la réforme ont été décrites. Ces résultats permettront de mettre en évidence les principales différences et convergences des trames argumentatives mobilisées par les anti-réforme et par les pro-réforme. Nous conclurons en soulignant quelques enjeux propres à l'élaboration d'un discours politique et militant.

\section{Approche cognitive de l'action publique : référents théoriques}

L'analyse des discours politiques et institutionnels est un moyen d'accéder aux grands types de rationalité ou grilles d'intelligibilité du réel qui sont en compétition, qui doivent être compris comme le cadre cognitif et normatif des actrices et acteurs et qui traduisent le « narratif» ou le « récit » de la cause pour laquelle elles et ils s'engagent (Radaelli, 2004). Celui-ci est tout à la fois contraint et contraignant, dans le sens poststructuraliste du terme. S'inscrivant dans une approche cognitive de l'action publique, des politologues se sont saisis de ces réflexions épistémologiques et de philosophie politique pour analyser dans le détail les formes de savoir (ancrage disciplinaire; types de connaissances mobilisés, algorithmes), les valeurs et normes d'action, ainsi que les images qui irriguent l'action publique (Muller, 2004; 2005; Roe, 1994). Ces éléments de discours traduisent le "référentiel» d'une politique sectorielle qui entretient un dialogue avec le référentiel global dominant d'une société. Ainsi, dans les sociétés occidentales capitalistes et dans le contexte du tournant néolibéral, tout actrice et tout acteur qui produit un discours politique et militant promeut une certaine vision du monde qui entretient un dialogue implicite ou explicite avec le référentiel de marché (Jobert et Muller, 1987; Muller, 2009) dans lequel les valeurs de responsabilité individuelle, les normes d'action d'efficacité et 
de performance tiennent une place majeure. Depuis les années 1980, ce référentiel s'est transformé et l'opinion publique en a été informée, notamment par les médias. Des penseuses ou penseurs du politique, des intellectuelles ou intellectuels et des chroniqueuses ou chroniqueurs relaient l'idée de la crise du néolibéralisme. Certaines et certains y voient une occasion de construire un nouvel ordre plus solidaire, par exemple, Rifkin ou Bollier; d'autres, comme Lordon ou Snowdon, y voient aussi l'espace propice au développement de tentations autoritaires. Si le néolibéralisme n'en est plus à son âge d'or et que les discours politiques des gouvernantes et gouvernants sont moins articulés autour du référentiel de marché pour légitimer des réformes, ce qui l'a remplacé est diffus.

L'explication des changements de politiques sociales est nourrie par une littérature foisonnante en science politique (Merrien, Parchet et Kernen, 2005). Elle est souvent présentée comme pouvant prendre trois directions : centrée sur les actrices et acteurs, sur les institutions ou sur les idées. Des chercheuses et chercheurs préconisent des recherches permettant de saisir simultanément ces trois niveaux d'analyse (Palier et Surel, 2005; 2010). L'explication de la transformation du modèle d'action publique en matière de soutien au revenu au Québec a été abordée par des recherches centrées sur les actrices et acteurs en termes, notamment, de répertoires d'action (Dufour, 2004; Noël, 2002). Plus souvent, les recherches scientifiques ou les enquêtes menées par des organismes communautaires ou en partenariat avec des milieux scientifiques ont davantage été descriptives. Ces politiques de soutien au revenu ont en effet été décrites en termes d'impacts pour les populations cibles, de conditions d'accès et de niveau des prestations financières, ainsi que d'organisation des services, institutionnels et communautaires, ou de mode de gouvernance (Groulx, 2009). Cette recherche fait partie des travaux qui s'inscrivent dans une démarche visant à élucider le rôle des «idées» dans les transformations de l'action publique.

Selon Muller (2004, p. 376), «un acteur ne pourra pas construire n'importe quel système de croyance en fonction de ses besoins stratégiques. Le référentiel d'une politique, ce n'est pas seulement des idées, mais des idées en action». La réforme récente de l'aide sociale a entraîné la mobilisation politique de nombreux actrices et acteurs qui dans leurs discours, ont opéré un choix stratégique parmi un ensemble de savoirs et valeurs disponibles ainsi que parmi des formes rhétoriques possibles. Celles et ceux qui diffusent leurs opinions et leurs connaissances ont tranché dans le réel en faisant des opérations de sélection des faits et de hiérarchisation des valeurs. Au plus près des débats citoyens ayant entouré la récente réforme de l'aide sociale, la question centrale fut pour nous de comprendre les «normes» (Muller, 2004), ou les «types de rationalité» en compétition 
(Pires, 2004). Dit autrement, quelle normativité apparaît comme dominante et quelle normativité se retrouve écartée? Notre travail empirique a très rapidement permis d'établir que par-delà les divergences d'opinion au sujet de la réforme, les références cognitives et normatives mobilisées concouraient à mettre en scène différents profils relativement stéréotypés et de prestataires qui pouvaient donc être traités comme des indices forts de la normativité plébiscitée. Quels personnages idéaux typiques sont mobilisés dans les articles qui cherchent à convaincre l'opinion publique de la légitimité ou de l'illégitimité de la réforme? Quels sont ceux qui ressortent majoritairement et ceux qui sont marginalisés?

L'intérêt de se centrer sur des discours médiatiques est d'accéder d'une part à ce qui est l'ordinaire des références normatives et cognitives auxquelles tout un chacun est fréquemment exposé et d'autre part, aux formes et contenus argumentatifs qui pour les actrices et acteurs qui les répandent, ont été identifiées comme pertinents pour sensibiliser un large public, voire le convaincre. Privilégiant une large source de discours, nous avons eu accès autant à des récits produits par des personnes habituées à l'expression dans les médias - présentant souvent une élaboration collective d'un narratif (par exemple, pour ceux qui représentent un organisme communautaire, un parti politique, ou une ligne éditoriale) - qu’à des récits de lectrices ou de lecteurs qui ne sont pas soumis à la contrainte de l'élaboration collective. Les actrices et les acteurs s'inscrivent cependant toutes et tous dans le même contexte socio-politique, avec sa rationalité dominante, qui vient contraindre leurs choix discursifs.

\section{Cadre méthodologique}

Les catégories conceptuelles de l'analyse cognitive de l'action publique ont balisé notre stratégie d'analyse (Muller, 2005), soit : les types de connaissance mobilisés (ancrage disciplinaire, statistiques, récits d'expérience, citations d'experts, de rapports ou ouvrages censés faire référence, algorithmes), les valeurs, les normes d'action, et les images qu'utilisent dans leurs articles les autrices et auteurs pour soutenir leur argumentation. Les valeurs sont des principes éthiques généraux, par exemple la "dignité humaine » ou la "justice sociale». Les normes d'action se rapprochent des valeurs puisqu'elles participent aussi à la normativité, mais elles s'en distinguent en tant que principes gouvernant directement l'action, telles les expressions types les introduisant : "il faut que... ", « il doit... elle doit / ne doit pas...». Elles viennent expliciter les valeurs mobilisées; sans elles, le contenu de ces dernières resterait flou, susceptible d'interprétations opposées; car on connaît bien la polysémie des valeurs précitées et leur mobilisation par des actrices 
et acteurs ayant pourtant des conceptions politiques opposées. Les algorithmes sont des relations causales simplificatrices entre deux situations et se traduisent aisément par l'expression : "Si... alors...", que l'on peut aussi traduire : "Si... = ... ». Les images sont quant à elles des raccourcis cognitifs permettant de saisir à leur seule évocation la grande ligne d'intelligibilité d'une situation, autrement plus complexe, que souhaite donner une autrice ou un auteur en utilisant une expression-choc: «elles sont des vecteurs implicites de valeurs, de normes ou même d'algorithmes; elles font sens immédiatement sans passer par un long détour discursif» (Muller, 2004, p. 372). Évidemment, il y a un saut à utiliser ces catégories construites pour l'analyse des débats qui se tiennent dans différentes arènes politiques et en particulier celui des espaces officiels et institutionnels, telles les arènes parlementaires ou les réunions ministérielles. Les médias n’offrent pas l'espace pour un discours en profondeur; nous n'accédons en ce sens qu'à des narratifs courts qui sont proches de ce que Muller nomme des «images». Le «récit imagé» (nous soulignons) nous paraît être la formule qui correspond le plus au matériau que nous avons collecté et analysé.

L'ensemble des articles d'opinion (tribunes politiques et militantes, tribunes d'éditorialistes, courrier des lecteurs) parus dans trois journaux de masse québécois ont été colligés : La Presse, Le Devoir et Le Journal de Montréal. Ce dernier occupe une place importante dans le secteur des quotidiens francophones. Il tranche de par la prépondérance de l'information locale et sportive, ses nombreuses photos, son style plus direct et accessible. C'est sans doute ce qui en fait un journal populaire dans tous les sens du terme. La Presse est le quotidien francophone le plus ancien. D'abord proche du pouvoir conservateur, il évolue rapidement pour se rapprocher des combats des classes ouvrières. À l'instar de quotidiens américains de son époque, il est connu pour être centré sur la nouvelle. Le groupe propriétaire de La Pressel'est également de six autres quotidiens, Le Nouvelliste, La Tribune, Le Soleil, Le Droit, Le Quotidien et La Voix de l'Est, qui sont disponibles en ligne sur cyberpresse.ca. Quant à lui, Le Devoirest un quotidien indépendant qui a la réputation d'avoir pour lectorat l'élite québécoise.

Notre corpus a été constitué à partir de la base de données Eureka; les mots clés suivants ont été utilisés : " aide sociale ", " assistance sociale ", " assisté social», déclinés en nombre et en genre. Il inclut les articles parus entre le $1^{\text {er }}$ janvier 2015 (le mois de janvier correspondant à l'annonce d'une réforme du programme de l'aide sociale) et le 31 décembre 2016 (un peu plus d'un mois après la promulgation de la loi). Du corpus volumineux colligé, nous avons distingué les articles d'information, d'enquête et d'opinion. Nous avons délibérément choisi de nous arrêter sur les articles d'opinion et 
d'en opérer un classement en fonction de l'étendue qu'y tient le thème de l'aide sociale : dominante ou minoritaire. Le corpus d'articles d'opinion où le thème de l'aide sociale est dominant est composé de 141 articles se répartissant ainsi : 92 articles parus dans La Presse, 24 dans Le Journal de Montréal et 25 dans Le Devoir. À notre connaissance, La Presse ne s'est pas davantage préoccupée de ce sujet; elle est un média qui produit généralement davantage de contenu. Notons aussi qu'elle inclut en ligne de nombreuses éditions régionales. Le tableau suivant présente les grands types d'actrices et acteurs qui se sont exprimés sur l'aide sociale entre janvier 2015 et décembre 2016 :

\section{TABLEAU 1}

\begin{tabular}{|l|c|c|c|c|c|}
\hline \multicolumn{1}{|c|}{$\begin{array}{c}\text { Acteurs } \\
\text { Journaux }\end{array}$} & $\begin{array}{c}\text { Lecteurs } \\
\mathbf{( N = 5 5 )}\end{array}$ & $\begin{array}{c}\mathbf{0 C} \\
\mathbf{( N = 4 8 )}\end{array}$ & $\begin{array}{c}\text { Éditorialistes } \\
\mathbf{( N = 2 3 )}\end{array}$ & $\begin{array}{c}\text { Politiques } \\
\mathbf{( N = 1 0 )}\end{array}$ & $\begin{array}{c}\text { Universitaires } \\
(\mathbf{N}=\mathbf{5})\end{array}$ \\
\hline La Presse & $\mathrm{n}=34$ & $\mathrm{n}=42$ & $\mathrm{n}=9$ & $\mathrm{n}=7$ & $\mathrm{n}=0$ \\
\hline Le Devoir & $\mathrm{n}=11$ & $\mathrm{n}=6$ & $\mathrm{n}=0$ & $\mathrm{n}=3$ & $\mathrm{n}=5$ \\
\hline Le JDM & $\mathrm{n}=10$ & $\mathrm{n}=0$ & $\mathrm{n}=14$ & $\mathrm{n}=0$ & $\mathrm{n}=0$ \\
\hline
\end{tabular}

Nous avons codifié manuellement l'ensemble du corpus. Au départ, la fréquence, les répétitions de certaines références normatives et cognitives nous ont donné quelques idées sur les enjeux du débat (Pires, 1997a); une analyse plus systématique a ensuite été faite afin de nous donner accès aux narratifs politiques des différentes personnes qui se sont exprimées. Cette description s'appuie sur les récits imagés qui sont produits sur les prestataires de l'aide sociale, l'État, les organisations responsables de l'administration du programme et la communauté dans laquelle s’inscrivent ces actrices et acteurs.

Nous avons par la suite procédé par stratégie inductive (Poupart et collab., 1997) en relisant notre corpus et en utilisant des catégories conceptuelles inspirées de l'analyse cognitive des politiques publiques. Ainsi, nous avons pu affiner les figures de prestataires mobilisées dans les récits imagés. Ces figures ne sont pas toujours transparentes; cependant, elles le sont bien davantage que dans les articles où le thème de l'aide sociale est minoritaire, et où notre tentative de reconstruire les traits caractéristiques du personnage de prestataire s'est révélée vaine. Dans ces articles, les prestataires apparaissent comme toute autre actrice ou tout autre acteur social vivant des situations de vie complexes à l'intersection de multiples processus sociaux et rapports d'oppression. Dans une démarche inductive, les interprétations ont été assurées par de multiples relectures, et par «triangulation des 
chercheu[se]s» (Pelletier et Pagé, 2002) et nous les avons mises à l'épreuve du corpus qui avait fait l'objet des opérations de classification précitées. Nous avons produit des synthèses par grands groupes d'autrices et auteurs (membres et représentantes ou représentants d'organisme communautaire, lectorat, élues ou élus politiques, éditorialistes, chercheuses ou chercheurs) et par positionnement au regard de la réforme : groupes partisans et opposants.

Quatre figures de prestataires émergent de notre corpus : celles de "parasite», de "paresseux », de «solidaire» et de "vulnérable». Les termes ont été choisis en fonction du signifié qui ressort de l'ensemble des indices colligés à partir des catégories conceptuelles précitées et non du souci d'utiliser les signifiants des actrices et acteurs. Ils permettent d'embrasser l'ensemble des situations décrites tout en permettant de les discriminer entre elles. La figure du parasite désigne la ou le prestataire qui profiterait du système au détriment d'autres membres de la société par l'usage de stratagèmes lui permettant de cumuler plusieurs sources de revenus, tandis que celle du paresseux désigne celle ou celui qui n'a pas acquis ou qui a perdu l'éthique de l'effort et qui se contente de vivre de l'aide sociale. La figure du vulnérable désigne la ou le prestataire dont le parcours s'expliquerait par des défaillances personnelles et familiales et par des structures socioéconomiques, culturelles et politiques injustes. Enfin, la figure de la solidarité désigne une ou un prestataire dont l'investissement en société se décline de manière alternative, soit par un investissement dans la sphère privée, soit par un investissement dans l'espace public (autre que la sphère économique).

\section{Les références normatives et cognitives des pro-réforme et des anti-réforme}

\section{Les «prestataires des anti-réforme»}

Les qualificatifs qu'utilisent les anti-réforme pour désigner les personnes visées par cette dernière sont : «faibles» «les plus démunis et plus souffrants dans la société », « les plus mal pris[e]s dans la société ", «les humiliés », «les quêteux», "les prestataires», «les allocataires », "les sans-emploi ", "des amies, mères, bénévoles et militantes", "un artiste à la merci de l'assistance sociale» et "des citoyens à part entière». Sans être complètement exhaustive, cette liste permet d'illustrer la diversité des termes employés; elle montre surtout que l'image que se font les anti-réforme n'est pas de teinte uniforme et varie selon leurs mises en scène de prestataires ou selon diverses histoires de recours à l'aide sociale. 
Les premières expressions citées dans cette liste, qui pointent la vulnérabilité des prestataires, ne sont pas en elles-mêmes représentatives de l'argumentaire mobilisé pour contester le bien-fondé de la réforme en matière d'aide sociale. D'autres indices discursifs doivent être relevés dans les articles.

En effet, chez beaucoup d'anti-réforme, l'ensemble de l'argumentaire tend plutôt à décrire les conséquences très concrètes de vivre avec peu de ressources financières. Ainsi, de brefs énoncés descriptifs souligneront que les prestataires ont recours «aux banques alimentaires», ont "une espérance de vie moindre» et une "santé fragilisée»; chez les universitaires ayant surtout intervenu dans Le Devoir, des références à des événements historiques, comme la "mobilisation citoyenne» ayant permis l'adoption en 2002 de la Loi visant à lutter contre la pauvreté et l'exclusion sociale ou le plan Marshall de 1949, des références conceptuelles seront ajoutées : "exclusion", "pauvreté», « désaffiliation ». Dans la même veine, un algorithme fréquemment mobilisé est le risque afférent au fait de venir réduire une prestation déjà insuffisante pour couvrir les besoins de base. La relation causale mise en perspective souligne que la mesure envisagée par le gouvernement est susceptible d'entraîner plusieurs risques de préjudices, et en premier lieu celui de l'itinérance traduit de manière imagée par le risque de se retrouver «à la rue». Est aussi avancé l'argument du cercle vicieux de la pauvreté en l'opposant plus ou moins explicitement au cercle vicieux de l'assistanat. Des formes rhétoriques plus dramatiques sont mobilisées : «éclabousser de son sang sur les murs», "payer encore plus de sa propre chair». À l'occasion de la veille de la Saint-Valentin, un auteur soulèvera par exemple que pour les prestataires, le fait de vivre en couple est difficile (Lecteur, Le Devoir), ou, encore qu'en Angleterre, des sanctions infligées aux prestataires seraient responsables de nombreux suicides (Éditorialiste, $\mathrm{La}$ Presse). Dans ces énoncés, il n'y a pas d'identification d'un profil type de prestataire; son histoire de vie n'est mise en scène qu'au travers la situation concrète de vivre avec peu de revenus ou de se voir menacé d'en avoir moins encore. Est donc pointée la vulnérabilité socio-économique dans laquelle vivent tous les prestataires.

En revanche, d'autres trames argumentatives qui pointent la vulnérabilité des prestataires mobilisent des références cognitives et normatives qui tendent à apporter une clé de lecture de leur situation relativement réductrice. En effet, elles et ils sont directement décrits comme n'ayant fréquemment pas «de diplôme ou un diplôme d'études secondaires" justifiant la nécessité de mettre en place des plans d'accompagnement "personnalisés» et "adaptés aux besoins». Plus radicalement, des autrices et auteurs reprennent le chiffre ministériel selon lequel «90\% des aptes ne le sont pas» (Représentante d'un organisme communautaire, La Presse). Ce même chiffre est repris à l'envers par un 
lecteur : «moins de $10 \%$ ont le potentiel d’intégrer le marché du travail». Dans le sillage de ce chiffre, seront évoqués en plus de l'insuffisance du parcours scolaire, des problèmes de santé mentale et de toxicomanie. La fermeture d'un centre pour personnes ayant un problème de consommation de drogues au mois de janvier 2016 charrie avec elle cette image de prestataires avec des vulnérabilités personnelles (même si l'on y pointe aussi que les choix politiques peuvent les aggraver). En affirmant qu'un «enfant qui naît dans un tel environnement [d'une famille bénéficiaire de l'aide sociale] sera marqué à jamais d'un stigmate», un éditorialiste du Journal de Montréals'opposant à la réforme met en scène des prestataires dont les itinéraires de vie se réduiraient à une forme de déterminisme social. Ce déterminisme est mis en scène avec la mobilisation du personnage de proche aidant, " des personnes qui peuvent vivre des situations difficiles en lien avec la maladie d'un proche, la garde d'enfants en bas âge, des problèmes de violence familiale». La vulnérabilité psychomédicale personnelle ou familiale est ainsi une sous-catégorie très mobilisée par les actrices et acteurs. Dans d'autres récits, on mobilisera davantage l'idée-ressource des structures vulnérabilisantes, par exemple lorsque des autrices ou auteurs pointent des phénomènes de discrimination sur le marché du travail. Tous ces argumentaires convergent pour souligner que le fait de recourir à l'aide sociale ne serait pas un choix, soit pour des raisons psychofamiliales, soit pour des raisons sociopolitiques.

À l'opposé, d'autres récits imagés font émerger des figures alternatives où la question du choix est abordée de manière plus nuancée. Ces figures alternatives apparaissent toutefois avec parcimonie dans l'ensemble de notre corpus; notre présentation des personnages de prestataires qui émergent des discours des opposants ne serait toutefois pas complète sans en faire état. Le principal personnage de ce type de récit est féminin : ce sont des femmes qui s'investissent dans ce que l'on appelle le care (soins des enfants, des personnes âgées ou ayant la charge d'une personne avec un handicap), le bénévolat et parfois le militantisme : «Les femmes assistées sociales sont d'abord et avant tout des mères, des amies, des bénévoles, des militantes, et leur plus grande réussite dans la vie ne sera sûrement pas d'être obligées d'accepter un programme d'employabilité». Il ne s'agit pas ici de pointer une vulnérabilité personnelle dans le fait d'être une femme dépendante de l'aide sociale, mais de souligner qu'il y a d'autres façons de mener une existence digne que celle du recours au travail rémunéré; "Môman travaille pas, elle a trop d'ouvrage", une autre expression de ce type de récit. Plus marginale encore, car elle n'émerge que dans Le Devoir, c'est celle de l'artiste. Il est par exemple mentionné que cela peut être un "choix raisonnable» que de recourir dans cette situation à l'aide sociale. 
Nous retrouvons dans tous les journaux des exemples de l'utilisation de références cognitives et normatives qui abondent à décrire des personnages de prestataires dans ces différentes formes mises en évidence.

\section{Les «prestataires des pro-réforme "}

Les pro-réforme utilisent quant à elles ou à eux les qualificatifs suivants pour désigner les personnes qui en sont visées : «bougons", «fraudeurs ", "récalcitrants», « résistants", «assistés sociaux». Ces qualificatifs négatifs ne sont pas fréquemment employés; ce sont davantage de courtes anecdotes personnelles et des images évocatrices qui sont évoquées. "Une poussée dans le dos", "mériter le chèque ", renvoient à la norme d'action punitive plébiscitée. Pour celles et ceux qui soutiennent le Projet de loi 70, la personne prestataire développerait un véritable "mode de vie qu' [elle] choisit sciemment ", "refuse l'aide au travail », estime que " tout lui est dû sans effort de sa part », vivant ainsi « dans le meilleur de deux mondes ». D'autres s'interrogent :

"Je ne sais plus quoi penser. Malheureusement, je connais beaucoup de gens qui vivent des prestations d'aide sociale et qui pourraient travailler, comme j'en connais qui n'ont pas le choix et qui ne peuvent vivre avec ce que le gouvernement leur donne. Comment trouver un juste équilibre?»

À travers ces exemples, c'est un personnage assez monochrome qui émerge : la malice ou la paresse dont il est affublé sert à condamner moralement les "personnes capables de travailler » et qui reçoivent néanmoins une prestation d'aide sociale.

Ces récits font apparaittre une ou un prestataire qui a le choix. Pour certains, l'argumentaire est aussi étayé par un algorithme selon lequel favoriser le "laisser-aller ", c'est risquer de mettre en péril les autres membres de la société, soit celles et ceux qui travaillent ou ont travaillé, "les honnêtes travailleurs contribuables " ou les "payeurs d'impôts de la classe moyenne». Le personnage de prestataire mis en scène dans ces récits imagés est celui de la personne apte, mais parasite et paresseuse. Évoquant l'expérience d'une directrice régionale d'Emploi Québec, le ministre accrédite lui-même l'existence d'un tel personnage :

"L'un des centres locaux d'emploi sur son territoire avait convoqué pas moins de 60 prestataires d'aide sociale [...]; le jour venu, il n'y a eu que cinq prestataires à la rencontre d'information et deux seulement se sont inscrits par la suite à des mesures de réintégration. " (La Presse, 14 juillet 2016) 
Ce récit d'expérience s'inscrit dans le recours aux mesures punitives (algorithme : si pas de mesures punitives $=$ non-participation aux programmes d'employabilité = persistance dans l'assistanat = non-retour sur le marché du travail). Les pro-réforme relaient également le cercle vicieux de l'assistanat ou le risque de la transmission intergénérationnelle. C'est là qu’une figure collective émerge : celle des «jeunes».

En mobilisant le personnage du jeune vulnérable qui n’a pas eu "grande chance dans sa vie», mais dont "l'indice d'employabilité est élevé ", le ministre met en scène une distinction entre inadaptées ou inadaptés réformables et inadaptées ou inadaptés qui ne le sont pas. Dans chacune des tribunes du ministre, la catégorie de "jeunes» fait une apparition, et ce, sous les traits de la vulnérabilité personnelle ou familiale. Le seul éditorialiste de La Presse à avoir défendu le projet de loi reprend l'argumentaire gouvernemental estimant que la mesure coercitive et punitive vise principalement des " jeunes de moins de 35 ans " pour justifier la nécessité de mesures de préemployabilité et d'employabilité. Toujours dans La Presse, de rares partisanes ou partisans de la réforme, intervenant comme représentantes ou représentants d'un organisme communautaire et de plusieurs organismes patronaux, évoquent aussi cette vulnérabilité en arguant que ces jeunes ont des problèmes de comportement et une famille dysfonctionnelle.

Chez les autres partisanes ou partisans de la réforme, des lectrices et lecteurs de $L a$ Presse ou des éditorialistes du Journal de Montréal, on retrouve également cette figure de jeune qui n'apparaît que très peu chez les opposantes ou opposants. Son emploi est davantage paradoxal : " jeunes chômeurs qui sollicitent pour la $1^{\text {ère }}$ fois l'assistance sociale "; " les jeunes doivent mériter l'aide "; «" Les deux seules personnes prestataires que je connais et qui ont moins de 35 ans... la première a un emploi saisonnier non déclaré et la $2^{\mathrm{e}}$ semble assez bien s'en sortir en déjouant habilement le système." »; « les jeunes dont 6200 sont issus de familles bénéficiaires de l'aide sociale "; " les jeunes de moins de 35 ans avec problèmes de comportement ».

Le blâme moral transparaît, la question de l'inadaptation psychosociale également. Quoi qu'il en soit, la référence discursive aux "jeunes», dont l'employabilité pourrait être améliorée, a soutenu peut et doit être prise en compte a soutenu la norme d'action punitive; les éditorialistes, représentantes ou représentants d'organisme de la société civile, lectrices et lecteurs, partisanes et partisans de la réforme la reprennent fréquemment dans leurs tribunes et courriers.

De tous les journaux consultés, le personnage de paresseuse, de paresseux ou de parasite n'apparaît pas dans Le Devoir, nous le retrouvons en revanche dans les deux 
autres journaux. Cependant, dans La Presse, les éditorialistes se distancient de cette représentation, lui préférant celle de la personne vulnérable, avec des défaillances.

\section{Groupes partisans et groupes opposants à la réforme : différences et convergences}

L'analyse des récits met au jour des valeurs en tant qu'objets de conflit d'appropriation. La dignité et la justice - pour ne citer que les plus populaires - sont revendiquées de manière attendue par celles et ceux qui s'opposent au projet de loi gouvernemental. En revanche, ses partisanes et ses partisans soulignent que ce discours axiologique de délégitimation de la réforme est non fondé. L'appartenance des prestataires à la commune humanité est cœur de la réforme. Pour les unes ou les uns, cette dernière relève de la responsabilité sociale envers tout individu et vient justifier un droit inconditionnel au secours, à un filet de sécurité sociale suffisamment généreux. Pour les autres, les prestataires, sans inaptitudes identifiées, sont capables de participer à l'effort collectif. Ainsi, chez un chroniqueur du Journal de Montréal (7 septembre 2016), une politique de soutien inconditionnel est jugée infantilisante pour les prestataires et la vulnérabilité est présentée comme une fiction qui nie la capacité de tout individu à avoir du pouvoir sur sa vie.

Chez les éditorialistes et le lectorat, il y a un arrimage relativement stable entre l'image que l'on se fait des bénéficiaires et le choix de l'orientation normative des dispositifs publics d'aide sociale. En effet, chez ces actrices et acteurs, la justification d'une approche punitive par les pro-réforme est associée au personnage de prestataire avec des traits de caractère associés à la malice ou la paresse, tandis que la justification d'une approche d'accompagnement, redistributrice et de droits sociaux inconditionnels est associée au personnage de prestataire affichant ceux associés à la vulnérabilité et, marginalement, à la solidarité. Notons que du côté des partisanes et partisans de la réforme, et du premier d'entre eux, le ministre, le personnage vulnérable, inadapté psychosocial, est aussi mobilisé, nous l'avons vu, dans leur stratégie argumentative. Cependant, peu d'interventions chez les partisanes ou partisans le rejoignent et le lectorat de son côté n'a pas retenu cette figure pour justifier la réforme; elle est davantage l'apanage des éditorialistes et de rares représentantes ou représentants d'organismes communautaires qui y sont favorables. Les anti- réforme, et en particulier les groupes communautaires, ont pu par leur choix narratif déconstruire les mythes de ses supporters (" les bougons "; " le BS assis sur son cul toute la journée »; « les maudits-BS-qu’on-fait-vivre-avec-nos-taxes-et-nos-impôts »), mais moins la trame argumentative de ses autrices et auteurs. 
Nous avons pu ainsi tracer les principales lignes de force autour des personnages convoqués : chez les anti-réforme, la personne vulnérable de ses défaillances personnelles et/ou la personne vulnérable socio économiquement du fait de ses conditions de vie et de facteurs macro et méso externes; chez le ministre, la personne paresseuse et présentant une vulnérabilité personnelle; chez les lectrices, lecteurs ou éditorialistes soutenant la réforme, la personne dite paresseuse ou profiteuse. Dans leur façon de présenter les prestataires, il y a donc bel et bien des différences majeures entre les groupes partisans et les groupes opposants à la réforme. Cependant, deux thématiques, celle de la vulnérabilité et de l'éthique du travail, nous permettront de pointer des convergences au sein de certaines trames argumentatives.

\section{La vulnérabilité personnelle}

La figure de la prestataire ou du prestataire vulnérable est largement empreinte de l'idée selon laquelle l'employabilité serait à parfaire, grâce à des programmes conçus par des spécialistes des besoins en main-d'œuvre non satisfaits et à des psychoéducatrices ou psychoéducateurs. Chez les groupes s'opposant à la réforme, elle n'est pas spécialement incarnée par les jeunes, mais d'autres personnages sont convoqués : les "toxicos", les "décrocheuses, décrocheurs", et les personnes ayant un problème de santé mentale, les mères monoparentales et les personnes proches aidantes.

Les cadres théoriques les plus fréquemment mobilisés dans les écrits pour comprendre les itinéraires des personnes dépendantes des systèmes d'aide publique et dites en difficulté sont ceux de la pauvreté, de la précarité, de l'exclusion sociale ou de la désaffiliation. Par exemple, nombre de personnes ont souligné que les travaux sociologiques qui se sont attardés à décrire les itinéraires des jeunes mettent davantage en scène des problèmes sociaux, des défaillances, des fragilités ou des anomies que la capacité pour eux de s'émanciper (Astier, 2009; Robert et Pelland, 2007).

Quasi unanimes dans leur valorisation de la responsabilité politique et des causes structurelles dans l'aggravation de la situation des prestataires, les détractrices ou détracteurs du modèle punitif mobilisent également fréquemment un stéréotype des personnes prestataires comme portant des vulnérabilités psychofamiliales et éducationnelles. Stratégie militante ou réelle interprétation réductrice basée sur des statistiques permettant d'identifier les principaux déterminants sociaux du recours à l'aide sociale, il ne nous appartient pas de trancher et notre enquête - qui est uniquement basée sur l'analyse documentaire et non sur des rencontres avec les militantes et militants - ne nous permet 
pas de le faire. En revanche, cette présentation, qui peut partiellement correspondre à la réalité, nous paraît devoir être questionnée puisqu'un principe de base de la sociologie est de ne pas confondre une corrélation avec un lien de causalité et qu'elle vient réduire la complexité des itinéraires de vie des personnes qui, à un moment de leur vie, ont eu recours à l'aide sociale.

\section{L'éthique du travail}

Dans les stratégies discursives mobilisées, le point largement aveugle est l'absence d'un discours déconstruisant l'idée selon laquelle vivre grâce au marché du travail va de soi et serait la norme désirable en soi, indépendamment du contexte social plus large dans laquelle les expériences au travail prennent un sens qui n'est pas toujours désirable, ni pour la personne (souffrance au travail), ni pour la société (travail comme destructeur de ressources environnementales, par exemple). Cette normativité est au contraire fortement irriguée, sauf rare exception, par des discours qui ne viennent pas mettre en cause le type d'emploi vers lequel cette personne vulnérable serait accompagnée et sans souligner les enjeux en termes de liberté individuelle d'être ainsi dépendante du marché du travail (McAll, 2009).

Si l'analyse des récits imagés du milieu communautaire et du lectorat qui s'opposent à la réforme met au jour chez les prestataires une situation traversée par une tension dialectique entre l'inadaptation issue de trajectoires personnelles et familiales complexes et l'existence de structures économiques et politiques inadaptées, très peu de récits font émerger son dépassement en mettant en scène un horizon de l'accompagnement dont la finalité ne serait pas de rejoindre le marché du travail rémunéré. Ce constat révèle ainsi que des actrices et acteurs - y compris celles et ceux du milieu communautaire — ne s'autorisent pas, ou que partiellement, à s’affranchir de la normativité dominante à l'œuvre dans cette réforme de l'aide sociale. Les figures du parasite, paresseux et de la vulnérabilité personnelle sont mobilisées pour servir une éthique du travail et de la responsabilité. Chez les groupes opposés, il y a que très peu de références normatives et cognitives qui abonderaient dans le sens d'une éthique de la sollicitude qui s'articulerait aux théories de la justice (Tronto, 1994).

De la façon dont elle est mise en scène, la vulnérabilité des prestataires est comprise dans deux grandes directions : psychomédicale, profondément inscrite dans les itinéraires individuels et familiaux (psychologisme dominant chez les éditorialistes de La Presse et chez les politiques promoteurs de la réforme) ou au contraire, profondément inscrite dans les 
itinéraires des groupes sociaux victimes d'injustices de redistribution et de reconnaissance (sociologisme, chez certaines représentantes ou certains représentants d'organismes communautaires). En ce sens, l'expérience des actrices et acteurs " prestataires » n'apparaît pas comme potentiellement en mouvement, transitoire, frappée par le hasard, ou encore, comme choisie. Quel que soit le média, de nombreux récits imagés mettent en scène des prestataires aux itinéraires stéréotypés, et cela pose selon nous des enjeux à la fois théoriques et pratiques pour la construction d'un discours politique et militant.

\section{Conclusion}

\section{Enjeux pour la construction d'un discours politique et militant}

Dans un contexte où les milieux politiques et militants s'interrogent sur la manière de réagir face aux formes dominantes de discours, un premier effort pourrait justement porter sur la mise au jour du cadre cognitif et normatif dominant qui irrigue une politique. Cela passe par l'analyse minutieuse du contenu des discours, des «idées " qui trament un modèle d'action publique. Si nous concevons avec Muller (2004, p. 376) que le référentiel global opère une contrainte et qu'un acteur ne peut "pas construire n'importe quel système de croyances en fonction de ses besoins stratégiques", selon la lecture poststructuraliste (Butler, 2002; Mouffe, 2000), il y a cependant des résistances discursives du "petit pas de côté", qui offrent la possibilité de s'affranchir d'une grille d'intelligibilité du réel dominante. En particulier, des lectrices, lecteurs, représentantes et représentants du milieu communautaire, de même que certaines ou certains universitaires, ont fait ce "pas" pour restituer des expériences alternatives positives ou complexes. Au contraire, la diffusion de ce personnage avec ses manques ou ses défaillances psychoéducatives se rapproche davantage d'un référentiel d' "investissement social» (Dufour et collab., 2007), dont la consonance plus douce que celle de «marché» n'est pas synonyme d'absence d'une autre forme de violence institutionnelle, même parée d'un discours centré sur la vulnérabilité personnelle et familiale. La mobilisation de ce personnage contribue à nier les facteurs structurels qui le créent et le renforcent, ou encore à contester l'existence d'itinéraires de vie alternatifs ou complexes.

Par ailleurs, l'éthique du travail qui est supporté par les récits autour de la paresse, du parasitisme, mais aussi de la vulnérabilité personnelle et économique, avec des normes d'action centrées sur l'accompagnement à l'insertion, apparaît une stratégie discutable. En effet, même si nous acceptons l'idée que les récits politiques et scientifiques ont toujours 
une part oppressive — dans la mesure où la grille d'intelligibilité du réel qui y est diffusée vient gommer la diversité des situations et les constitue en retour comme moins riches —, la nature et l'intensité de cette oppression sont cependant variables. Nous ne nous pensons qu’à travers les représentations disponibles. Certaines expériences minoritaires ou encore certaines expériences de groupes minoritaires n'accèdent que peu à la visibilité. Le sujet en construction ne peut donc s'en saisir et y puiser des références pour mener son travail de réflexivité sur lui-même. Vivre avec peu de revenus « renvoie à des expériences protéifomes". Or, mobiliser des récits simplificateurs en les diffusant explicitement ou en adhérant implicitement à la fiction d'un personnage stéréotypique comme étant vulnérable signifie que les actrices et acteurs concernés ne peuvent s'y reconnaître. Cela peut les éloigner de la participation politique et surtout les empêcher de se reconnaître dans leur choix de vie — dans la majorité des cas plus transitoires qu'enracinés — ou encore dans des destins tragiques où le hasard tient un rôle. Le nœud principal nous apparaît être celui de la qualification ou de la disqualification de certaines expériences de vie afin que celles-ci ne soient pas réifiées dans des récits qui peuvent apparaître paternalistes (les groupes partisans de la réforme) ou maternalistes (certains groupes opposés à la réforme). Offrir la possibilité aux actrices et acteurs d'articuler un discours ambigu sur leurs expériences et d'en penser la complexité est un point d'horizon de la construction du discours politique et militant inclusif. Il y a assurément plus d'émotions à aller chercher dans ces histoires de vie, et potentiellement rejoindre un large public1, qu'à mobiliser des catégories sociologiques désincarnées qui objectivent le phénomène de recours à l'aide sociale, en énumérant des figures stéréotypiques de "décrocheuses ", de «décrocheurs", de " toxicomanes » ou de " mères monoparentales ».

Nos analyses finales indiquent que les figures mobilisées au départ traduisent de manière imparfaite certaines ambivalences du discours et d'autres distinctions directrices ont émergé (distinction entre les prestataires qui sont adaptables au marché du travail et celles ou ceux qui ne le sont pas) qui permettent de circonscrire davantage les enjeux et les défis pour la construction d'un discours politique et militant.

Une des limites de notre approche se rapporte au type de média composant notre corpus. Nous avons privilégié des journaux plutôt que des médias audiovisuels et les réseaux sociaux où s'expriment d'autres types d'intervenantes ou intervenants. Nous aurions sans doute eu accès à plus de jeunes, à plus de diversité en termes de classe sociale également. Un prolongement de cette recherche serait celui de tenter d'interpréter la place de cette figure de la vulnérabilité ou de l'inadaptation psychosociale comme grille d'intelligibilité importante dans ce débat. Mener un travail de comparaison historique 
des articles d'opinion parus lors de la réforme importante de l'aide sociale de 1989 et qui révélerait, par contraste, que la figure dominante était alors celle de la parasite ou du parasite, de la paresseuse ou du paresseux, pourrait être un indice que le référentiel néolibéral est transformé, voire remplacé. C'est une hypothèse que nous émettons, et qui serait évidemment à vérifier. Mais cette stratégie comparative (Soulet, 2011) pourrait alors expliquer en partie pourquoi des actrices et acteurs s'opposant à la réforme ont construit leur trame argumentative en diffusant parfois explicitement ou implicitement la fiction d'un personnage stéréotypique que ne renieraient pas les autrices et auteurs de la réforme.

\section{Bibliographie}

ASTIER, Isabelle (2009). "Les transformations de la relation d'aide dans l'intervention sociale», Informations sociales, Vol. 2, № 152, p. 52-58.

BUTLER, Judith (2002). La vie psychique du pouvoir, Paris, Léo Scheer Éditions, 309 p.

COLLECTIF POUR UN QUÉBEC SANS PAUVRETÉ (2016). Portrait des personnes à l'aide sociale : données statistiques et paroles citoyennes, réf. du 25 avril 2019, http://www.pauvrete.qc.ca/ document/portrait-personnes-a-laide-sociale-donnees-statistiques-paroles-citoyennes/

DUFOUR, Pascale, et collab. (2007). «L'investissement social au Canada. Emergence d'un référentiel global sous tension ", dans Olivier Giraud et Philippe Warin (dirs.), Politiques publiques et démocratie, Paris, La découverte.

DUFOUR, Pascale (2004). «L'adoption du projet de loi 112 au Québec : le produit d'une mobilisation ou une simple question de conjoncture politique ?", Politique et sociétés, Vol. 23, Nos 2-3, p. 159-182.

ESPING-ANDERSEN, Gøsta (1999). Les trois mondes de l'État-providence. Essai sur le capitalisme moderne, Paris, PUF, 308 p.

FRETEL, Anne, et collab. (2018), «Éditorial. Contrôler les chômeurs : une histoire qui se répète (forte de ses croyances et à l'abri des réalités)", Revue française de Socio- Économie, Vol. 20, No 1, p. 9-25.

GROULX, Lionel-Henri (2009). «La restructuration récente des politiques sociales au Canada et au Québec : éléments d'analyse», Labour/Le Travail, 63, p. 9-46.

IRIS (2012). Les prestations d'aide sociale sont-elles trop généreuses?, réf. du 25 avril 2019, http://irisrecherche.s3.amazonaws.com/uploads/publication/file/Note-Aide-sociale-FINAL-web-02.pdf.

JETTÉ, Nicole, et Sylvia BISSONNETTE (2012). "L'aide sociale de 1961 à 2012, tours de vis ", Revue du CRÉMIS, Vol. 5, № 2, p. 40-46. 
JOBERT, Bruno, et Pierre MULLER (1987), L'État en action : politiques publiques et corporatismes, Paris, PUF, $242 \mathrm{p}$.

McALL, Christopher (2009). " De l'individu et de sa liberté », Sociologie et sociétés, Vol. 41, № 1, p. 177-194.

MERRIEN, François-Xavier, Raphä̈l PARCHET et Antoine KERNEN (2005). L'État social : une perspective internationale, Paris, Armand Colin, 362 p.

MOUFFE, Chantal (2000). «Féminisme, citoyenneté et démocratie plurielle», dans Thanh-Huyen Ballmer-Cao, Véronique Mottier et Lea Sgier (dires.), Genre et politique : débats et perspectives. Paris, Folio essais, № 370, p. 167-197.

MULLER, Pierre (2000). «L'analyse cognitive des politiques publiques: vers une sociologie politique de l'action publique», Revue française de Science Politique, Vol. 50, № 2, p. 189-207.

MULLER, Pierre (2004). «Référentiel», dans Laurie Boussaguet, Sophie Jacquot et Pauline Raviner (dires.), Dictionnaire des politiques publiques, Paris, Les Presses de Sciences Po, p. 555-562.

MULLER, Pierre (2005). "Esquisse d'une théorie du changement de l'action publique ", Revue française de Science Politique, Vol. 55, No 1, p. 155-87.

MULLER, Pierre (2009), Les politiques publiques, Paris, PUF, 128 p.

NOËL, Alain (2002). «Une loi contre la pauvreté : la nouvelle approche québécoise de lutte contre la pauvreté et l'exclusion sociale», Lien social et Politiques, № 48, p. 103-114.

PALIER, Bruno, et Yves SUREL (2010) Quand les politiques changent: temporalités et niveaux de l'action publique, Paris, L'Harmattan, $422 \mathrm{p}$.

PALIER, Bruno, et Yves SUREL (2005). «Les « trois i » et l'analyse de l'État en action », Revue française de science politique, Vol. 55, No 1, p. 7-32.

PELLETIER, Céline, et Ginette PAGÉ (2002). Les critères de rigueur scientifique en recherche. Méthodologie de recherche en soins infirmiers, № 68, p. 35-42.

PIRES, Alvaro (1997a). « De quelques enjeux épistémologiques d'une méthodologie générale pour les sciences sociales», dans Poupart, et collab. (dirs.), La recherche qualitative. Enjeux épistémologiques et méthodologiques, Montréal, Gaëtan Morin Éditeur, p. 3-54.

PIRES, Alvaro (2004). « La recherche qualitative et le système pénal. Peut-on interroger les systèmes sociaux ", dans Dan Kaminski et Michel Kokoreff (dirs.), Sociologie pénale : système et expérience, Toulouse, France, Érès, p. 173-198.

POUPART, Jean, et collab. (dirs.) (1997). La recherche qualitative. Enjeux épistémologiques et méthodologiques, Montréal, Gaëtan Morin Éditeur, 405 p.

RADAELLI, M. Claudio (2004). «Récits (policy narrative) », dans Laurie Boussaguet, Sophie Jacquot 
et Pauline Raviner (dires.), Dictionnaire des politiques publiques, Paris, Les Presses de Sciences Po, p. 548-554.

ROBERT, Marie, et Marie-Andrée PELLAND (2007). "Les différentes postures à l'égard du travail salarié chez des jeunes vivant en situation de précarité : Subir, résister et expérimenter ", Nouvelles pratiques sociales, Vol. 20, No 1, p. 80-93.

ROE, Emery (1994). Narrative Policy Analysis, Theory and Practice, Durham, Duke University Press, $240 \mathrm{p}$.

SOULET, Henry (2011). «Interpréter, avez-vous dit!», Régimes d'explication en sociologie, réf. du 25 avril 2019, http:// journals.openedition.org/sociologies/3471

TRONTO, Joan (1994), Moral Boundaries. A political Argument for an ethic of Care, New York, Routledge, 242 p.

\section{Note}

1 Puisque l'histoire qui nous a le plus rejointes est celle de Jeanne racontée par Suzanne Lafontaine (La Presse, 24 juillet 2016) : «Retour à la case départ. Incapable de tenir dans pareille impasse financière, Jeanne perd son logement et se retrouve à la rue. À 61 ans. Jeanne, c'est vous, c'est moi. Il n'y a pas ici d'histoire de drogue, d'alcool, de criminalité, de problème de santé mentale. Rien. Juste la vie ordinaire». 